\title{
La frontera sinaítica de Egipto. Del 'mapa' de Seti I a la fotografía satelital
}

\author{
Liliana M. Manzi* \\ María Violeta Pereyra *
}

\begin{abstract}
MANZI, L.M.; PEREYRA, M.V. La frontera sinaitica de Egipto. Del 'mapa' de Seti I a la fotografia satelital. Revista do Museu de Arqueologia e Etnologia, São Paulo, 20: 293-310, 2010.
\end{abstract}

Resumo: Compara-se a informação geográfica representada nos relevos militares de Seti I, em Karnak, com a geomorfologia do norte do Sinai para elucidar as práticas iconográficas do Reino Novo e discutir a homologação recorrente na bibliografia dos assentamentos arqueológicos localizados na área com as fortalezas mencionadas naquele monumento.

Para isso, procedeu-se à análise do registro epigráfico e das unidades geomorfológicas a partir de imagens de satélite, o que permitiu: precisar o sentido das cenas; estabelecer com precisão quais foram os terrenos disponiveis durante o Reino Novo em um ambiente altamente dinâmico; e discutir o papel dos assentamentos de fronteira.

Palavras-chave: Iconografia - Mapa de Seti I - Karnak - Relevos militares Fronteira do Sinai - Unidades geomorfológicas - Assentamentos fortificados.

\section{Introducción}

E 1 empleo de las expresiones iconográficas del arte monumental del antiguo Egipto como fuentes para la reconstrucción de su historia nos enfrenta a dos cuestiones iniciales conexas: la naturaleza de las fuentes y el alcance de nuestra interpretación.

La investigación de la frontera del estado faraónico y las relaciones con el Levante se encuentra asi sujeta a la interpretación de la evidencia, que en el caso de los materiales epigráficos apela de manera enfática a los instrumentos creados por los historiadores del

(*) CONICET; Universidad de Buenos Aires. <violetapereyra@filo.uba.ar>, <lm_manzi@yahoo.com.ar> arte para su decodificación. ${ }^{1}$ En nuestra discusión de los relieves militares de Karnak de Seti I, adoptamos una perspectiva que considera las reflexiones realizadas en otros campos del conocimiento para escudriñar los significados no evidentes de la frontera oriental alli representada, que tomamos como evidencia.

Ubicados en el exterior de la sala hipóstila, en el lado este de la pared norte del gran templo de Amón, estos relieves forman parte de una composición que comprende otras escenas

(1) En la presente investigación asumimos que los monumentos del culto divino y su decoración constituyeron expresiones poderosas del discurso social faraónico y fueron creados como parte del sistema de control social por la monarquía. 
militares. ${ }^{2}$ A lo largo del muro se registraron las acciones del faraón contra diferentes pueblos. Nuestra secuencia narra la campaña del año 1 de Seti I a Pa-Canaán y muestra el desplazamiento del soberano en su carro en un territorio que se identificó con el camino real que conectaba Egipto con el sur de Palestina: el 'Camino de Horus' (Gardiner 1920: Figs. 11 y 12). También se ha sugerido que la serie de fuertes alli representados correspondia a los asentamientos del estado egipcio que durante el Reino Nuevo jalonaban la costa norte de Sinai.

Esta representación es conocida como el 'mapa' de Seti I (Fig. 1) y desde su publicación por Alan Gardiner en 1920 se sucedieron diferentes estudios en los que se pretendió interpretar los eventos que la escena describia para reconstruir la secuencia de acciones militares que habria emprendido a comienzos de su reinado ${ }^{3}$ e identificar los lugares representados en el relieve con los sitios arqueológicos descubiertos en la franja norte de Sinaí.

Nuestro objetivo en este trabajo es mostrar que esta escena fue elaborada, igual que muchas otras de su tipo, para exaltar el poder real sobre fundamentos míticos. Las acciones militares del soberano exhibidas en ese discurso fueron un medio para hacerlo en un contexto de gobierno con fuerte sustento militar, ${ }^{4}$ por lo que una homologación con la evidencia arqueológica es posible sólo recreando los nexos analógicos y anacronismos entre los asentamientos fortificados del norte de Sinaí.

(2) Las campañas representadas del lado oeste del muro son: en el registro inferior a Pa-Canaán, en el registro medio una expedición contra Yenoam y Líbano, en tanto que las escenas del registro superior se perdieron. Del lado este se representaron: en el registro inferior una campaña contra los heteos, en el medio una contra los libios y en el superior contra Qadesh y Amurru (Degrève 2006: 48-49 y Pl. VIII-XII)

(3) Los más destacables son los de Breasted (1906: 37-76), Gardiner (1920: 99-116), Faulkner (1947: 34-39), Gaballa (1976: 100-105), Spalinger (1979: 29-47 y 2005), Broadhurst (1989: 229-234), Murnane (1992) y El-Saady (1992: 285-294)

(4) Los gobernantes fueron de origen militar a partir del reinado de Horemheb, cuyo acceso al trono representa un desplazamiento de otros grupos de poder que habian dominado la politica egipcia durante la dinastia 18 .
El recurso fue empleado como núcleo argumental para mostrar la identidad esencial del soberano, encarnación de las fuerzas ordenadoras del cosmos en continua renovación. La victoria mitica sobre el caos hace de Horus un eterno vencedor contra toda oposición que se le haga y Seti I es representado exactamente como el Horus triunfador sobre sus oponentes (Fig. 2).

Los asentamientos representados en el 'mapa' de Seti I evocan un sistema de ocupación de la franja norte de Sinaí que en la época se caracterizaba por la articulación de pequeños campamentos estacionales distribuidos en torno a una fortaleza o estación central que operaba como base de la red administrativa conocida como 'Camino de Horus' (Oren 1987). Los relieves sólo muestran la estructura central de los sitios, asociados a una fuente de agua, y la figura del soberano en su carro como ejecutor de la victoria.

El texto retórico, que sintetiza la acción propiamente dicha, aclara que en su primer año de reinado ${ }^{5}$ el rey destruyó a sus enemigos "desde la fortaleza de Sile hasta Pa-Canaán" y que trajo de regreso prisioneros con su carga de riquezas (Kitchen 1993: 8-9).

\section{El registro epigráfico y su articulación monumental}

A partir de la naturaleza compleja de nuestras fuentes, cuya epigrafia tiene un fuerte acento en lo icónico (Tefnin 1984: 55-56), es conveniente considerar en forma previa a nuestra discusión su carácter simbólico y el alcance de lo que las mismas denotan y connotan.

En primer lugar, las escenas que discutimos se encuentran asociadas con inscripciones jeroglificas que completan y precisan su sentido, no a la manera de meros epigrafes o aclaraciones, sino integradas con la representación para articular un lenguaje complejo. ${ }^{6}$ El empleo de elementos figurativos e inscripciones en la

(5) Definido como de "renacimiento" (Kitchen 1993: 9).

(6) Sobre la cuestión véanse Tefnin (1984 y 1991), Bryan (1996) y Angenot (2002). 

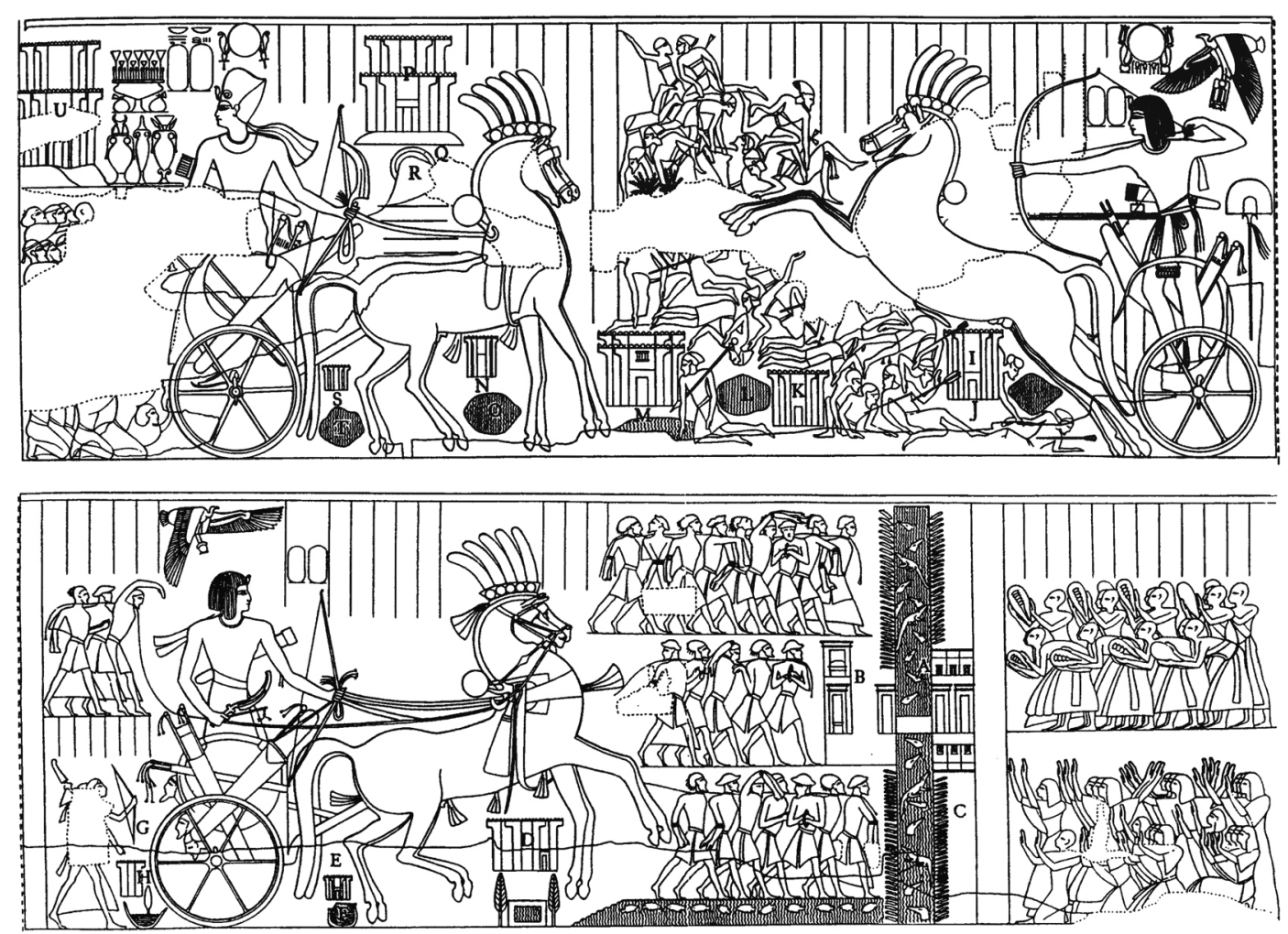

Fig. 1. El 'mapa' de Seti I (muro externo norte, lado este). a. Sección este (Gardiner 1920: fig. 11); b. Sección oeste (Gardiner 1920: fig. 12).

\begin{tabular}{|c|c|c|c|c|}
\hline Asiáticos honran a & $\begin{array}{c}\text { Triunfos de Seti I en } \\
\text { Seti I en su carro }\end{array}$ & $\begin{array}{c}\text { Regreso de Seti I con } \\
\text { prisioneros shasu }\end{array}$ & $\underset{\sigma}{\sigma}$ & $\begin{array}{c}\text { Recepción del } \\
\text { soberano } \\
\text { triunfante }\end{array}$ \\
\hline
\end{tabular}

Fig. 2. Esquema de los temas representados en el 'mapa' de Seti I.

composición de las escenas parietales de los templos corresponde a su funcionalidad simbólica, que trasciende el discurso racional para expresar la sacralidad que gobernaba la sociedad egipcia antigua.

En tal contexto, la creación de imágenes portadoras de significados se constituyó en instrumento apto para transmitir ideas, cuyos destinatarios - los miembros de la elite egipcia - podian decodificar. Una cuidada selección de escenas, figuras y motivos se aplicó para componer los textos que incorporaron palabras como parte de su estructura, aunque el mensaje podia ser entendido inclusive por contemporáneos iletrados. Es decir que el arte parietal egipcio comunicó ideologia con escritura y/o sin ella, ${ }^{7}$ e inclusive se registraron discordancias entre lo representado y lo escrito, siendo el último un signo evocador de prestigio (Bryan 1996: 167). Por eso, en la construcción del discurso literario se escogieron vocablos aptos para expresar ideas en consonancia con el discurso mítico implícito en las escenas, pero que no lo

(7) Bryan enfatiza la lectura incluso diferente de los mensajes, de acuerdo a la capacitad de los individuos de poder leer o no los textos jeroglificos (1996: 163-164). 
reiteraban. En el caso del 'mapa' de Seti I, se trata de un relieve cuya temática es el triunfo del faraón frente a sus enemigos, en el que textos retóricos y epígrafes de las figuras se conjugan para construir un retrato del soberano que exaltaba su aptitud para la victoria.

Como espacios definidos y sacralizados, los templos constituyeron recintos demarcados con claridad para ser diferenciados del espacio exterior, de carácter profano (Vergnieux 1989: 65-171). Su diseño respondió a las necesidades del culto, pero también a exigencias de carácter socio-político. Si se analiza una temática especifica de la decoración de un templo, como es nuestro objetivo para el caso de los relieves de Karnak, una cuestión preliminar será entonces la identificación de su localización y de sus relaciones con los restantes espacios arquitectónicos constitutivos del monumento. Así, el 'mapa' de Seti I necesariamente deberá ser considerado como parte de la temática general de la pared en la que fue integrado y ésta puesta en relación con el monumento en su totalidad, con el objeto de asegurar la lectura de todos sus niveles (Tefnin 1991: 66-87) y la comprensión de su estructura y semántica.

En primer lugar, la tripartición arquitectónica del templo egipcio diferenciaba ámbitos de carácter público, semipúblico y restringido, que expresaban una diferenciación a tener en cuenta, a la que se agrega la disposición de la decoración en las paredes interiores o exteriores, su orientación geográfica y su localización respecto de sus ejes (van Esschez-Merchez 1992: 216-220). Una arbitraria fragmentación del material epigráfico representa asimismo un cercenamiento de las relaciones funcionales entre los diferentes elementos decorativos del monumento, que resulta en una alteración de su significado, lo mismo que la interpretación de los elementos figurativos como evidentes (Tefnin 1979: 219-220).

Con respecto a la localización topográfica de los relieves militares de Seti I en Karnak, podemos atribuir una amplia accesibilidad al patio y los espacios abiertos, en tanto que el interior de la sala hipóstila, como espacio de mayor restricción, estaba abierto sólo a quienes participaban en los rituales. A cada sector correspondia una función cultual, siendo también de relevancia los significados politicos y sociales de las celebraciones ${ }^{8}$ y el emplazamiento de los relieves en el exterior de la sala hipóstila permite inferir que sus destinatarios eran sujetos cuyo acceso al interior de la misma estaba vedado.

En cuanto a la localización de la escena, está al norte con relación al eje principal del templo ${ }^{9}$ y dispuesta en el registro inferior del lado externo del muro. Según vimos, se la integró con otras escenas militares ${ }^{10} \mathrm{y}$ de interacción con el dios del imperio ${ }^{11}$ (Figs. 3 y 4).

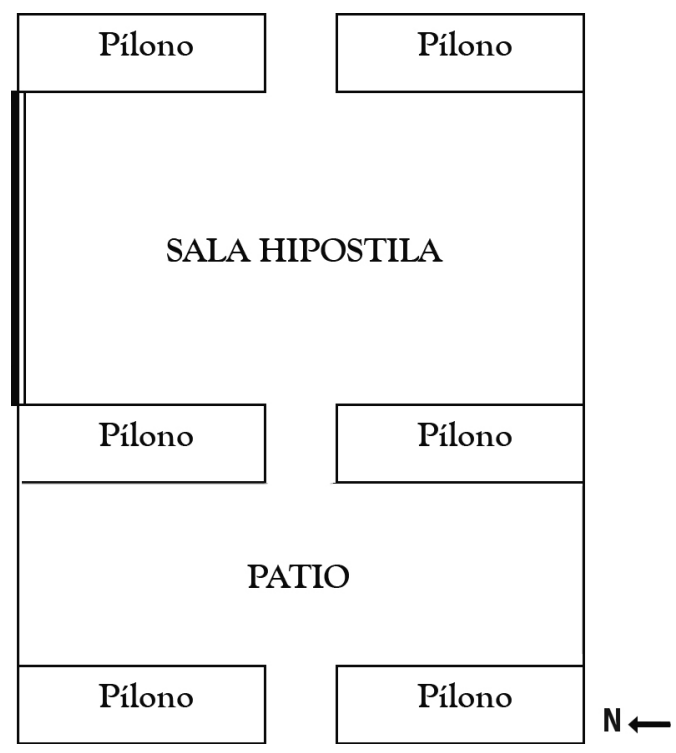

Fig. 3. Localización de la escena en la sala hipóstila del gran templo de Amón.

(8) El interés de los funcionarios por mostrarse como testigos de algunos rituales reales celebrados en los templos fue estudiado por Schulman (1988).

(9) No obstante su complejo proceso de construcción y reformas en el tiempo, el de Karnak es un templo axial cuya orientación oeste-este se articula con las vías procesionales (Cabrol 2001) empleadas en la celebración de la Bella Fiesta del Valle y la navegación por el Nilo. Su eje norte-sur se corresponde con otro sistema de vias procesionales que lo vinculaba con los templos de Khonsu y de Mut en Karnak y con el de Luxor.

(10) Dirigidas contra Tjehenu, Yenoam, Libano y Hatti.

(11) Representaciones de entrega de botin a Amón y de triunfo real. 


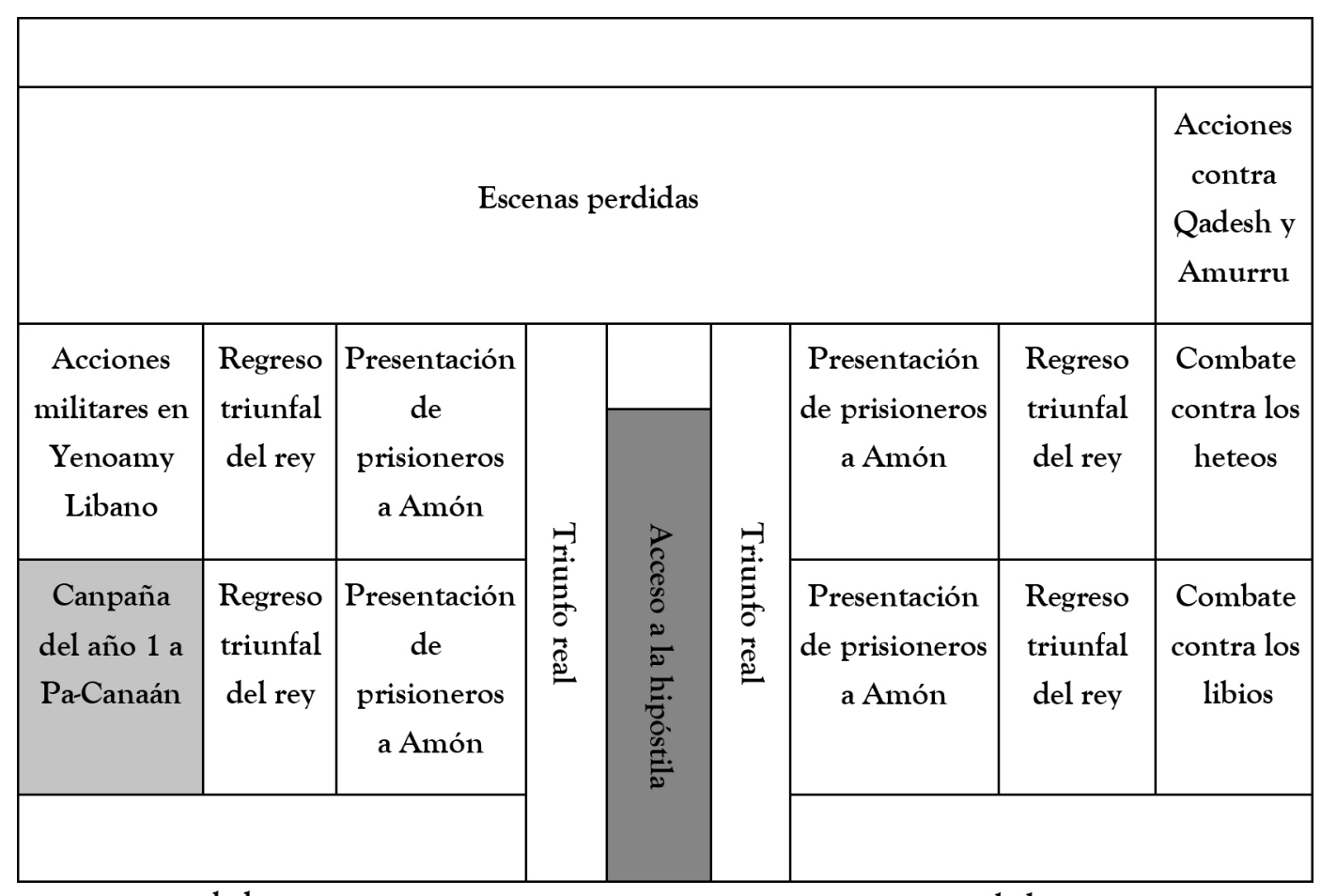

lado este

lado oeste

Fig. 4. Ubicación de la escena en el muro norte (lado externo) de la sala hipóstila.

El esquema de la Fig. 4 muestra la ubicación de las distintas escenas en el muro, dispuestas en registros, a ambos lados de la puerta que da acceso a la sala hipóstila desde el norte. Se puede observar alli que las representaciones de acciones militares se dispusieron hacia los extremos del muro, en tanto que el regreso triunfal del rey y la presentación de los prisioneros capturados al dios Amón convergen hacia las dos escenas de triunfo real que enmarcan el espacio de comunicación con la sala hipóstila (Degrève 2006: 48-49).

\section{El concepto de frontera}

El antiguo concepto egipcio de 'frontera' sea como 'limite' (preciso y lineal) o como 'borde' (impreciso o zona indefinida) requiere de una indagación en torno a su uso (Quirke 1989), pero sobre todo del reconocimiento del marco ideológico en el que las fuentes que los documentan encuentran su contexto de interpretación. Tal noción definiria en todo caso al territorio hasta donde el estado llegaba con su control, más allá del cual se encontraba el mundo externo, extranjero y desordenado que reconocemos como periferia de Egipto (Zingarelli 2003: 341-342).

Por otra parte, de acuerdo a sus fundamentos míticos el estado egipcio asumió una idea de sí mismo en la que se reconocía como un espacio social y politico delimitado y ordenado en contraste con el espacio exterior abierto y caótico. En este sentido, en época faraónica la frontera definía un área de encuentro entre un espacio sacralizado - que estaba bajo control del estado - y otro desordenado - dominio de individuos y fuerzas amenazadoras del orden.$-^{12}$

La legitimidad de tal definición la ofrecen las propias fuentes egipcias en las que se

(12) Lo que justificó la función esencial del soberano: mantener el orden primordial y re-crearlo cuando era alterado. 
documentan los términos $t 3 \breve{S}^{13}$ "frontera" ${ }^{14} \mathrm{y}$ $\underline{d} r / \underline{d} r w$ " 15 "borde", "limite", "fin", ${ }^{16}$ estando este último vinculado con la preposición "desde" (Gardiner 1979: \$176). Así, la idea de centralidad a partir de la cual se definia el movimiento hacia la periferia era expresada en egipcio por el vocablo $\underline{d} r w$ y en congruencia con la concepción de un soberano que "hace", "extiende" y "estira" las fronteras de su reino según su voluntad, ${ }^{17}$ como un quehacer que es expresión ostensible de su poder.

\section{La frontera oriental}

La mayor parte del norte de Sinaí es una llanura arenosa con pantanos de sal y aguas subterráneas salobres que recibe escasa lluvia y sólo tiene vegetación dispersa en la superficie. La principal fuente de agua de Egipto era el Nilo, cuya actividad regia las condiciones de la vida social y económica y, en consecuencia, las posibilidades de organización del estado. En la antigüedad las condiciones naturales del área diferian de las actuales no sólo por el clima sino también por la existencia de un brazo del rio que en época ramésida alcanzaba el Mediterráneo en Pelusium, ${ }^{18}$ al este del moderno Canal de Suez (véanse Figs. 6).

(13) Determinado con el signo jeroglifico que indica división ( $Z_{9}$ de la 'lista de Gardiner').

(14) Erman y Grapow traducen: „die beiden Länder abgrenzen (bei der Teilung Ägyptens zweischen Horus und Seth)" (1971: V 236) (Hr t3̌s t3wy en la teologia menfita por ejemplo). También „die Grenzen” ( $t 33$. $w)$, „der beiden Länder abgenzen" (1971: V 236). Faulkner da "boundary" (1976: 294).

(15) A menudo también $i \underline{d} r$ (Erman y Grapow 1971: V, 585).

(16) „Ende, Grenze” (Erman y Grapow 1971: V, 585);

"boundary, end, limit" (Faulkner 1976: 323-324).

(17) Las fuentes escritas a partir de la dinastia 12 se refieren al faraón como quien "hace" (ir), "establece" $(s m n)$, "impone" $(s r w d)$, "protege" $(m k)$ y "extiende" (swsh) las fronteras. Quirke (1989) reconoció los vínculos existentes entre el desarrollo de politicas egipcias expansivas a partir del Reino Medio y su formulación en términos de acciones personales del soberano desde el momento en que aparece una fraseologia imperialista. (18) Denominación conservada en el nombre de la moderna población de Baluza.
La distribución de los sitios en el noroeste del Sinai sugiere que el brazo Pelusíaco no alcanzó esa región antes del siglo XIII a.C. y, en consecuencia, el proceso de ocupación habria acompañado la dinámica geomorfológica del territorio asociada a la actividad del cambiante curso del Nilo (Marcolongo 1992: 24).

Los asentamientos en ese territorio contaron con una mezcla de población nativa y egipcia hasta el advenimiento de la dinastia 1 , cuando el estado faraónico por primera vez introdujo cambios para establecer en el Delta oriental un sitio estatal: Tell Ibrahim el-Awad. ${ }^{19}$ Sin duda, esto se relaciona con el establecimiento de limites claros a la jurisdicción del estado dinástico y la necesidad de reforzar su control sobre ellos. Tell Ibrahim el-Awad operó como apoyo para el tráfico y las expediciones militares hacia la periferia oriental de Egipto y fue servido por un centro religioso que, según Belova, era expresión del poder real en su frontera (2003: 121).

Sin embargo, una asociación simbólica más potente entre la realeza y la frontera surgió recién durante el Reino Medio, cuando diversas expresiones describen la actividad expansiva del rey en sus fronteras y se hacen frecuentes en la fraseologia real. Esto significaba que las acciones del faraón, que eran simbólicas de su poder, daban legitimidad y fundamento a la realeza. ${ }^{20}$ Como resultado de ello, el extremo oriental del Delta fue descrito en las fuentes reales egipcias como un espacio destinado a ser protegido y puesto bajo control estatal.

Los 'Caminos de Horus' se mencionan por primera vez en conexión con la frontera oriental en el Reino Medio ${ }^{21}$ y se corresponderian con la ruta representada en nuestro relieve del templo de Karnak. La propia denominación del

(19) Conjuntamente con otros importantes centros como Abidos, Hierakonpolis y Elefantina en el Alto Egipto. (20) En los Anales de Amenemhat II en el templo de Ptah por ejemplo, se registro el flujo de bienes suntuarios desde el exterior por obra del soberano durante el periodo de su corregencia con su predecesor (Altenmüller y Moussa 1991: 1-3)

(21) En los Anales menfitas de Amenemhat II (w3tt-hrr) y en el Cuento de Sinuhe (w3wt-hr). 
área como camino real recordaba la divinidad del rey-Horus que ejercía su control sobre ella.

En la dinastia 18 el objetivo del imperialismo tutmósida fue asegurar la circulación de bienes de prestigio entre el Levante y Egipto, para mostrar que el gobernante del "centro", ordenado y sacralizado, tenía control sobre la periferia, siempre caótica. Los cambios geopoliticos verificados en la región a fines de la dinastia $18^{22}$ y su potencial amenaza al dominio egipcio de Palestina llevaron a las campañas asiáticas que Seti I hizo registrar en el exterior de la pared norte de la sala hipóstila de Karnak, ${ }^{23}$ como testimonio de su aptitud para el triunfo y para celebración de la divinidad que lo conducia, ${ }^{24}$ puesto que fueron registrados como parte de sus múltiples acciones victoriosas sobre el extranjero.

El hecho de estar representado el rey en tres diferentes situaciones en el tratamiento de su campaña a Pa-Canaán y contra las tribus shasu indica que los relieves de Seti I se propusieron desarrollar el tema en por lo menos tres núcleos narrativos, a modo de secciones o capitulos de un texto que relataba las acciones reales conducentes a la afluencia de botin hacia el interior de Egipto.

En relación a las discordancias existentes entre los textos jeroglificos y las figuras representadas, es destacable que en tanto que los primeros hacen referencia a los prisioneros capturados y a "su carga" en varias ocasiones, las representaciones la omiten. La inscripción relativa al botin presentado a Amón dice: "Los jefes de los paises extranjeros están prisioneros y (cargan) sobre sus espaldas su tributo de toda clase de vasos preciosos de sus tierras (hechos) de plata, oro y lapislázuli verdadero, por el valor que tú (Amón) me diste sobre todos los paises extranjeros" (Kitchen 1993: 8-9).

(22) Que condujeron a la guerra de Amka bajo el reinado de Akhenaton.

(23) Y en otros monumentos contemporáneos como la primera y segunda estelas de Beth Shean, las estelas de Karnak, de Tiro, de Tell Nebi Mend y de Tell esh-Shihab, además de las listas topográficas (Degrève 2006: 47-48).

(24) También Tutmosis III grabó los anales de sus campañas en el gran templo de Amón en Karnak.
Una explicación a la omisión en el tratamiento figurativo de la victoria de Seti I puede encontrarse en los receptores del texto. Se ha señalado que conjuntamente con $t 3 \breve{s}$ "frontera" las fuentes egipcias expresaban la aptitud dominadora del soberano con el vocablo $n h t$, que podemos traducir como "fuerza" y "victoria" (Galán 1995). Detrás de tal uso lexicográfico se enmascaraba la idea de un triunfo siempre asociado a la obtención de botin y riquezas y, en consecuencia, Galán consideró que ésta era la forma egipcia de expresar el concepto moderno de 'imperio' (1995: 160). ${ }^{25}$ Los beneficiarios del botin fueron sin duda los miembros de la elite, destinatarios a la vez del mensaje contenido en las inscripciones.

No obstante, la disposición del 'mapa' en un espacio semipúblico permitia a los observadores iletrados conocer las empresas triunfales de su soberano, el Horus entronizado cuya fuerza se imponía sobre sus enemigos para dar protección a Egipto, e informaba a quienes podian leer las inscripciones acerca del rico botín capturado.

Por último, aún cuando los trabajos de excavación se encuentren en curso en la franja norte de Sinai, los indicios de existencia de un centro simbólico del poder en la frontera a comienzos de la dinastia 19 fueron hallados en Tell el-Borg, sitio en el que el registro arqueológico permitió identificar bloques inscriptos pertenecientes a un templo de Ramsés II (Hoffmeier 2004). La relevancia del hallazgo, sin embargo, no es suficiente para asegurar que el sitio estaba en la ruta descripta por los relieves de Seti I. Asimismo, la reconstrucción del trazado del Camino de Horus puede intentarse a partir de datos espaciales más seguros que la simbólica representación del rey. La intemporalidad del poder de triunfo de Seti hace sin duda sospechosa la precisión de la información que pueda ofrecer la escena, que no obstante debe hacer referencia a circunstancias

(25) En la lengua egipcia antigua no se conocen otros términos que puedan traducirse por "imperio", o "imperialismo". 
y lugares históricamente reales, aún cuando su interés principal fuera la evocación de contenidos de orden mitico.

El Camino de Horus, por lo tanto, puede buscarse con mayores probabilidades de éxito en las modernas representaciones de la geografía del norte de Sinai y las fotografias satelitales, que al permitir reconocer distintos rasgos geomorfológicos antiguos pueden servir mejor como evidencia para su trazado.

\section{El proceso de consolidación del paisaje en el norte de Sinaí}

La configuración del actual paisaje en el sector noreste del delta del Nilo y de la costa norte de la peninsula de Sinai (Fig. 5) es parte de un proceso geomorfológico altamente dinámico, que tuvo lugar a través del Pleistoceno tardio hasta el presente. En el transcurso del mismo se produjeron sucesiones biológicas que derivaron en ambientes con predominio fluvial, terrestre y marino.

\subsection{Los agentes geomorfológicos}

Los agentes geomorfológicos que contribuyeron a la consolidación de esos ambientes y paisajes comprenden:

a) fluctuaciones eustáticas del nivel del mar, referidas a transgresiones y regresiones marinas cuya evaluación es realizada en comparación con el actual nivel marino;

b) neotectonismo, relacionado con fallas tectónicas, es responsable del desplazamiento de placas y de la acumulación de sedimentos sobre el litoral marino, dando como resultado el avance del continente a expensas de mar - progradación - y de la modificaron de los sistemas de drenaje fluvial;

c) transporte de sedimentos fluviales, relacionado con cambios paleo-climáti$\cos$, que se manifiesta a través de secuencias estratigráficas en donde se observan las diferencias entre la carga y la composición sedimentaria acumulada; d) erosión costera, que deposita y redistribuye sedimentos de diversas génesis - marinas, fluviales y terrestres mediante el accionar del oleaje y de las corrientes marinas; y

e) actividad humana, que contribuye a la acumulación y remoción de sedimentos mediante la apertura y el mantenimiento de canales artificiales de riego y navegación, entre otras modificaciones posibles - por ejemplo la salinización de suelos -.

A partir de las secuencias litoestratigráficas disponibles, fueron graficados por Stanley y Warne (1998) mapas paleogeográficos que reconstruyen los cambios ocurridos en el paisaje (Fig. 5) que contribuyen a la discusión de las estrategias de uso del espacio durante el Reino Nuevo.

Entre el 35.000 y el 18.000 antes del presente, el espacio ocupado por el delta actual era una planicie aluvial cruzada por una serie de canales activos (Fig. 5A) (Stanley y Warne 1993, 1998). Los sedimentos que representan ese momento comprenden lodos de coloración marrón, principalmente de derivados del rio Nilo. Posiblemente se trató de un espacio conformado por depresiones comparables a las que en la actualidad bordean distintos brazos del Nilo y que resultaban periódicamente inundadas.

En este momento, el nivel del mar, a causa del accionar de movimientos eustáticos, se ubicó unos $125 \mathrm{~m}$ por debajo del nivel actual haciendo que la linea de costa estuviera unos $50 \mathrm{~km}$ al norte (Abu-Zeid y Stanley 1990; Abdel Wahab y Stanley 1991).

Entre el 15.000 y el 8.000 antes del presente, durante la transgresión flandriense, el nivel del mar subió y la línea de costa migró nuevamente hacia el sur (Fig. 5B) (Stanley y Warne 1998). Este momento está representado en la secuencia estratigráfica por lodos de color grises de origen fluvial y marino (Stanley 1988). Se registró, además, la depositación de arenas pleistocénicas sobre superficies holocénicas, que dieron origen a dunas transgresivas que se 
depositaron cuando el mar avanzó sobre la plataforma continental (Stanley 1988; Stanley y Wingerath 1996).

Hacia el 7.500 antes del presente comenzó la formación del delta a partir de un ambiente influenciado por: el mar que registraba movimientos eustáticos, los cuales motivaban que su nivel se ubicara entre 15 y $12 \mathrm{~m}$ por debajo del actual (Stanley 1988); procesos acrecionales, que contribuyeron a la acumulación de sedimentos a razón de 1 a $7 \mathrm{~mm} / \mathrm{año}$ (Stanley y Warne 1998); y neotectonismo, que condujo a que los desbordes del cauce principal fueran encauzados a través de canales excavados por la erosión fluvial sobre una amplia llanura que se extendía hasta la laguna Manzala, discurriendo con sentido noreste (Abu-Zeid y Stanley 1990; Abdel Wahab y Stanley 1991).

En torno al 6.500 antes del presente (Fig. 5C), el nivel del mar se ubicó entre 9 y 10 m por debajo del nivel actual, haciendo que el gradiente del río fuera más pronunciado. La morfologia del delta estuvo básicamente controlada por el canal Sebenítico, que transportó grandes volúmenes de arenas costeras conformando una extensa playa de acreción, en donde se alojaron lagunas poco profundas y marismas, en cuyas márgenes se depositaron formaciones de dunas casi

Fig. 5. Área de estudio y evolución geomorfológica del delta del Nilo (tomada de Stanley y Warne 1998) A, B, C, D, E.

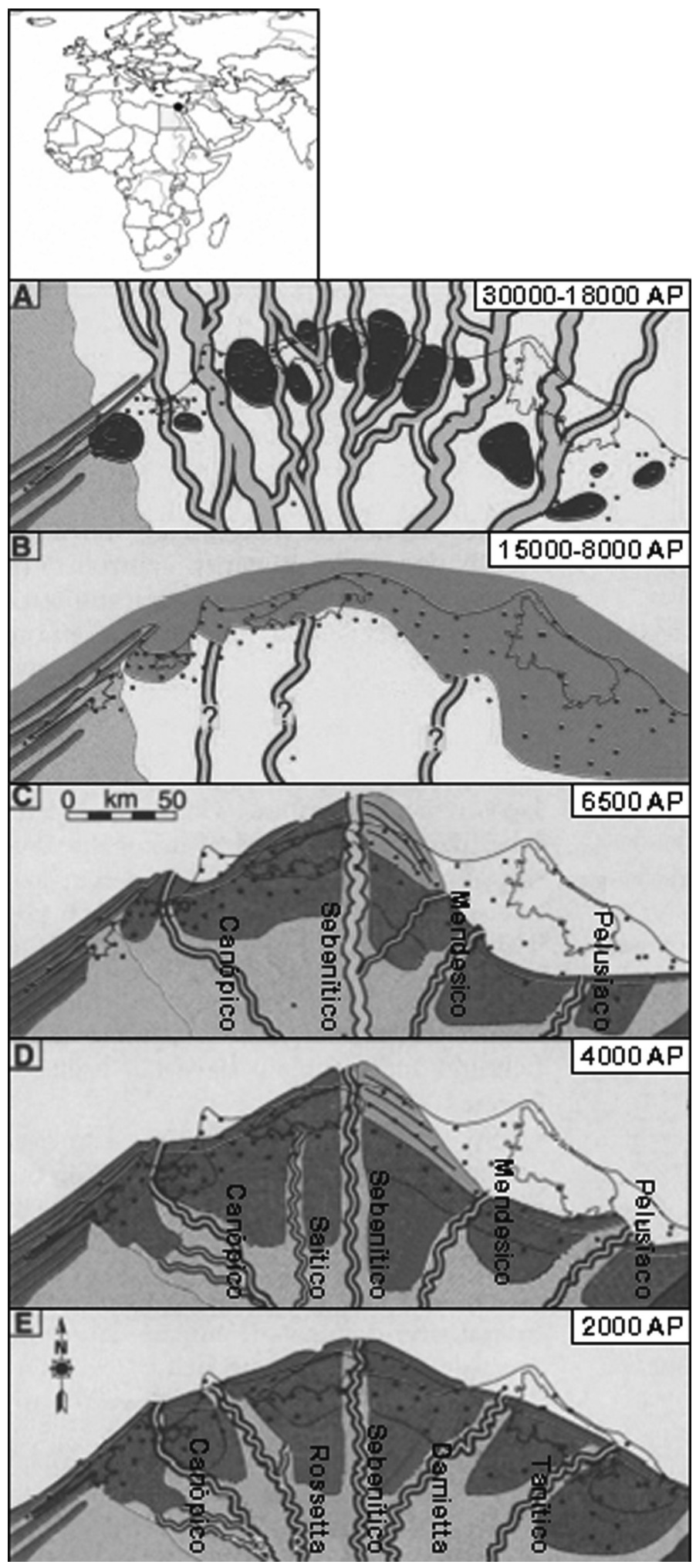


continuas (Stanley y Warne 1998). Al mismo tiempo, los vientos predominantes del este contribuyeron a la depositación de dunas modernas en el interior, desde Sinaí hasta el norte de Israel (Stanley y Wingerath 1996).

Unos 4.000 años antes del presente, el nivel del mar continuó ascendiendo lentamente, llevando a que el gradiente de la planicie del delta disminuyera (Fig. 5D) (Stanley y Warne 1998), haciendo que la acumulación de sedimentos aumentara y rondara en torno a unos $10 \mathrm{~mm} / \mathrm{año}$ en el sector noreste del delta (Stanley 1988), que continuó progradante, mientras que la costa norte y central comenzó a retrogradarse (Stanley y Warne 1998).

En el 2.000 antes del presente (Fig. 5E), el nivel del mar aumentó unos $2 \mathrm{~m}$, aunque se mantuvo por debajo del nivel actual. Las corrientes marinas y el oleaje modelaron el litoral costero. En este momento comenzó a configurarse el moderno delta, registrándose el predominio de ambientes fluviales (Stanley y Warne 1998).

En torno a los 1.000 años antes del presente, los mayores canales tributarios del Nilo se redujeron a dos, los brazos Rosetta al noroeste y Damietta al noreste y distintos promontorios de acreción se depositaron en sus desembocaduras a causa del transporte de sedimentos hacia la costa (Stanley y Warne 1998).

En la actualidad, la planicie del delta está limitada por acumulaciones de arenas y valvas depositadas por una corriente litoral prevalente de oeste a este, a lo largo de las distintas lineas de costa. Los fechados obtenidos sustentan antigüedades comprendidas entre los 1925 años antes del presente ( 25 d.C.) para las dunas más antiguas y los 345 años antes del presente (1.605 d.C.) para las más recientes (Sneh y Weissbrod 1973).

\subsection{Unidades geomorfológicas detectadas en tiempos históricos}

La descripción de las unidades geomorfológicas que configuraron el paisaje sobre el que se desarrollaron las actividades humanas desde el Reino Medio hasta la invasión persa tiene particular interés, al permitir evaluar la forma en que distintas porciones de terreno quedaron disponibles para ser ocupadas por poblaciones humanas.

El punto de partida lo señala la transgresión flandriense, cuya máxima expresión habria sido alcanzada hace unos 8.000 años antes del presente, momento en que las aguas del mar inundaron grandes extensiones de tierras interiores. El limite del área sumergida es reconocido en las imágenes satelitales como un cordón litoral fósil, depositado al norte de las lagunas oriental y occidental (Fig. 6a).

Al norte del cordón litoral y a causa de procesos eustáticos, tectónicos y sedimentarios se conformó una planicie de acreción constituida por depósitos con sedimentos marinos, fluviales y terrestres. Sobre esta planicie se observan distintas líneas de costa, también identificadas a partir de la depositación de cordones marinos con distintas cronologias, que fueron atravesados por canales tributarios del $\mathrm{Nilo}$, algunos de los cuales se secaron.

En fuentes documentales de distintas épocas son mencionados nueve brazos del Nilo, de los cuales seis se desecaron como consecuencia de la dinámica geomorfológica del delta. Solamente los brazos Rosetta y Damietta se mantienen activos. Entre ambos, los paleocanales de los brazos Tanítico, Sebenítico, Mendésico, Saitico y Atribico (Sneh y Weissbrod 1973) drenaban hacia el mar por la porción centro-norte del delta. El brazo Canópico discurria hacia el Mediterráneo al oeste del Rosetta y el antiguo brazo Pelusiaco, por su parte, mientras su curso se encontraba activo atravesaba en su recorrido el sector oriental del delta, al este del brazo Damietta (Figs. 5C y D). A partir del canal principal del brazo Pelusíaco se abrian sobre la margen norte dos tributarios menores. Luego del proceso acrecional costero la ubicación del paleocauce se ubicó al sudeste del mencionado canal actualmente activo (Fig. 6a).

El trazado de esta antigua cuenca de drenaje se encuentra en la actualidad bordeado por dunas que sepultaron los bordes estructurales del canal, que se manifiesta como una depresión de unos $25 \mathrm{~km}$ de largo, cuyo ancho varía entre 
80 y $100 \mathrm{~m}$. Este brazo estuvo relacionado con un canal artificial detectado al sur del mismo (Sneh y Weissbrod 1973) y cuyo trazado puede vincularse con los segmentos de un canal identificados al sur de la laguna oriental (Hoffmeier 2006).

La desaparición del brazo Pelusíaco es atribuida al accionar de movimientos tectónicos registrados en la llanura inferior del delta, que modificaron el sistema de drenaje e hicieron posible que se acumularan sedimentos que terminaron colmatando el canal y sus tributarios (Stanley; Bernasconi; Jorstad 2008). El momento critico de este proceso acrecional, a causa de la agradación costera, se produjo alrededor del 25 d.C. (Sneh y Weissbrod 1973).

Por último, resta mencionar que muy próximo a la costa del Mediterráneo se localiza el extremo este de la laguna Manzala, un cuerpo de agua de 1 a $2 \mathrm{~m}$ de profundidad que se originó por el hundimiento progresivo de la cuenca - proceso de subsidencia -. Las formaciones de dunas registradas en sus márgenes tienen edades holocénicas, siendo las acumulaciones australes más antiguas que las septentrionales, mientras que los promontorios Damietta y Port Said, y los cordones litorales que la separan del actual litoral marino son de formación reciente (Stanley 1988).

\section{La imagen satelital como soporte geográfi- co-ambiental de la representación iconográfica}

Las escenas del registro epigráfico que comprende el 'mapa' de Seti I parecen hacer directa alusión a una serie de elementos distribuidos en el paisaje.

La evolución del litoral marino del Mediterráneo oriental fue graficada reconociendo sobre la imagen satelital 36-30 N, LANDSAT 1990-2000, color artificial, http:// zulu.ssc.nasa.gov.mrsid, las unidades geomorfológicas identificadas por Marcolongo (1992) sobre imágenes satelitales Spot HRV 1988. A partir de este mapeo fue posible reconocer y evaluar cómo habría quedado conformado ese paleoambiente desde la transgresión flandriense.
La consolidación del paisaje natural durante el Reino Nuevo (ca. 3.200 antes del presente) resulta de interés para considerar las estrategias de ocupación del espacio, tanto en lo que respecta a la selección de los lugares de emplazamiento de los asentamientos como de las circunstancias que llevaron a su abandono final; la ubicación relativa de la linea de costa, del brazo Pelusíaco, de los canales artificiales y de las lagunas interiores debieron ser tenidos en cuenta al momento de ocupar y transitar ciertos lugares a expensas de otros.

Algunos de esos rasgos también fueron los utilizados por Hoffmeier $(2004,2006)$ al sostener que Tell el-Borg podría corresponder a La Morada del León, mencionada en los relieves de Seti I ${ }^{26}$ por ser el único asentamiento de envergadura que además presenta evidencia de construcciones fortificadas, luego de Hebua y Tell el-Herr.

\subsection{El paisaje natural como escenario de las ocupaciones humanas}

En ambientes desérticos como el norte de Sinai, la ocupación del espacio sólo es posible en relación a lugares con provisión de agua. De modo semejante, el tránsito terrestre debió estar en función de la existencia de cuerpos hidricos, sin mencionar que la circulación fluvial estuvo, necesariamente, condicionada por la existencia de brazos y canales tributarios del Nilo.

El tráfico marino sólo era posible uniendo puertos distribuidos a poca distancia unos de otros a lo largo de la costa que conformaron puntos estratégicos de comercio. En consecuencia, las travesías intermedias fueron cubiertas por medio de la navegación fluvial y del tránsito por rutas de caravanas.

En consonancia con el panorama planteado, la ubicación del antiguo brazo Pelusiaco se torna relevante por tratarse del único curso que atravesaba el extremo oriental de la planicie del delta, a diferencia de lo observado en los

(26) Identificado con la letra $\mathrm{D}$ en la publicación de Gardiner (1920: fig. 12). 
sectores centro y occidental, en donde se registraron por lo menos otros siete brazos.

Las investigaciones de campo realizadas por Sneh y Weissbrod (1973) estuvieron orientadas a partir de una serie de supuestos que, con algunas modificaciones, son presentados a continuación: 1. los documentos antiguos se refieren a un sector del espacio que podria ser identificado sobre el terreno; 2. el curso del brazo Pelusíaco debió estar en proximidad con asentamientos humanos; 3 . los canales de drenaje se distribuyen y articulan dentro de un sistema hidrográfico, por lo que sus trayectorias no son al azar; y 4. los paleocauces de rios y sus tributarios pueden ser reconocidos a través de algunos rasgos morfológicos distinguibles en trabajos de campo.

Los resultados alcanzados por estos autores les permitieron ubicar in situ el trazado del brazo Pelusíaco y reconocer, asimismo, los dos tributarios que se abrian sobre la margen norte, en dirección a la paleoplaya del Mediterráneo.

El paleopaisaje que se fue consolidando y modificando desde el Holoceno temprano comprendia una planicie de inundación del delta que era atravesada con dirección estenoreste por el brazo Pelusiaco del Nilo, formado en tiempos precedentes. El cordón litoral fósil, cuya ubicación se encuentra en el extremo sur de la mencionada planicie, marca el limite de los espacios inundados durante la transgresión flandriense (ca. 8.000 años antes del presente). Es posible que durante ese lapso la laguna oriental, que en la actualidad se localiza al sur de aquél, quedara rebasada por las aguas del mar adoptando la forma de una bahía, tal como se observa en el mapa (Fig. 6b) construido con información publicada por Hoffmeier (2006).

Poco después de alcanzado el punto más alto de la transgresión marina, las aguas comenzaron a retirarse y a dejar expuestos terrenos hasta entonces sumergidos. En consecuencia, el curso del brazo Pelusíaco se abrió sobre esa planicie de acreción en continua evolución, cuya ampliación llevó a que este tributario del Nilo prolongara su trayectoria, migrando hacia el norte en busca de su salida al mar.
Hacia fines del Segundo Periodo Intermedio (ca. 4.000 años antes del presente), el accionar conjunto de movimientos eustáticos, neotectonismo y procesos de agradación sedimentaria, inició el proceso de desecamiento del brazo Pelusiaco, que llevó a su desaparición siglos después del Reino Nuevo, hace unos 2000 años antes del presente.

\subsection{El paisaje cultural como factor de control efectivo e ideológico}

El proceso de ocupación de espacios en el extremo nororiental de Sinai se sustenta en el potencial habitacional que ofrecen ciertos terrenos, tales como posibilidad de saneamiento, cercanía a recursos naturales y acceso a vías de comunicación.

Los asentamientos de Hebua, Tell elGhaba, Kedua y Tell el-Borg comparten la caracteristica de presentar construcciones fortificadas asignables al Reino Nuevo y haber sido abandonados al finalizar el periodo Saita (Oren 1984: 13), probablemente como consecuencia de la invasión persa, momento al que corresponde el nivel más antiguo de ocupación de Tell el-Herr.

En el Reino Nuevo este sector de Sinaí se constituyó como un espacio de frontera en donde se registró evidencia de circulación humana e intercambio de bienes, a la vez que se constata la existencia de estructuras defensivas. Estas últimas son las que llevaron a distintos autores a interpretar que algunos de estos asentamientos de frontera corresponden a los representados en el 'mapa' de Seti I y que desde Gardiner (1920) hasta la fecha (Hoffmeier 2006) algunos arqueólogos se esforzaran en homologar lo alli representado con la geografia regional.

Los asentamientos de Hebua, Tell elGhaba y Kedua están emplazados sobre el antiguo cordón litoral depositado durante la transgresión flandriense, conformando un sector elevado dentro de la planicie del delta, mientras que Tell el-Borg y Tell el-Herr se ubican en cercanías de formaciones de dunas que rodean la margen sur de la laguna oriental (Fig. 6a). 

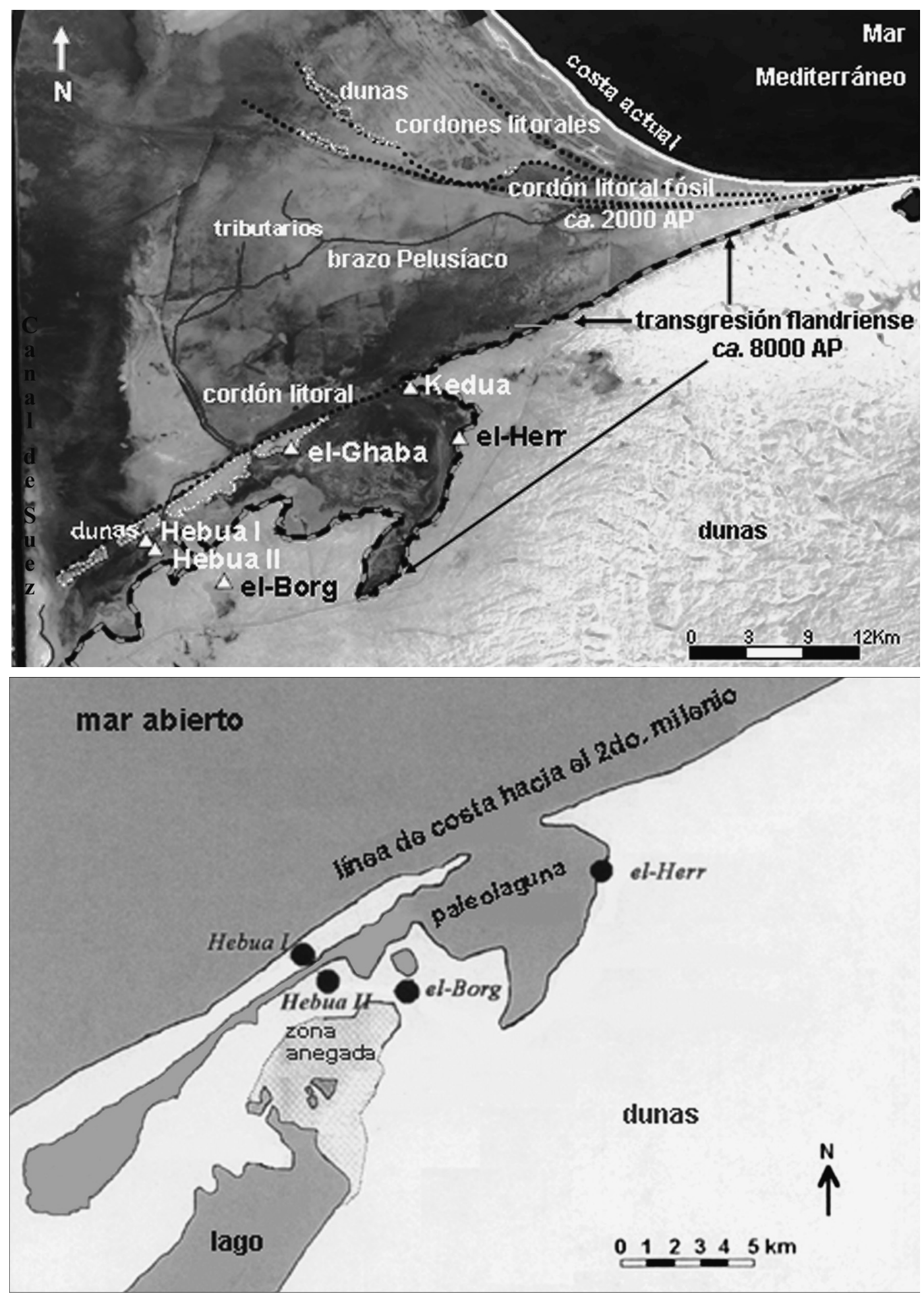

Fig. 6. El sector nororiental del delta del Nilo. a. Geomorfologia; b. Reconstrucción paleoambiental del sector noroccidental del delta del Nilo de acuerdo a Hoffmeier (2006). 
La localización de los asentamientos, en lo que respecta a las ocupaciones correspondientes al Reino Nuevo, no está en relación directa con la disponibilidad de tierras emergidas, como se desprende de la propuesta de Hoffmeier y elMaksoud (2003: 173), puesto que durante ese periodo, la costa del Mediterráneo se ubicaba varios kilómetros al norte de esos sitios. Por lo tanto, es muy probable que el emplazamiento de los mismos deba atribuirse a la continuidad ocupacional de un mismo sector del espacio y a un plan estratégico del estado que tuvo fines defensivos y que se materializó a través de la distribución de asentamientos con diferente complejidad estructural y arquitectónica, lo que seria concordante con las funciones cumplidas por cada uno de ellos en un área de frontera.

La propuesta de Hoffemeier $(2004,2006)$ ofrece una explicación analógica que trata de homologar el marco geográfico-ambiental de Tell el-Borg con las referencias geográficas contenidas en el 'mapa' de Seti I. Hoffmeier y el-Maksoud (2003: 195) señalan que un hipotético brazo del Nilo pasaba en su trayectoria por Tell el-Borg y desembocaba en una paleolaguna. Esta situación, a su entender, explicaba la inexistencia de murallas alrededor de los Fields II y III, dado que el curso hídrico habria actuado como defensa natural del templo y de las áreas funerarias alli identificadas.

Durante, las excavaciones en Tell el-Borg se registró la presencia de sedimentos de coloración marrón oscuro, a 3,15 m de profundidad, que fueron interpretados como pertenecientes a aquella rama del Nilo tempranamente desaparecida (Hoffmeier 2004, 2006). Pero, de acuerdo con la sección litoestratigráfica que da cuenta del proceso de formación del delta, se tiene que sedimentos de coloración semejante fueron identificados como depósitos fluviales acumulados por el Nilo, de edad pleistocénica, fechados entre el 35.000 y 18.000 antes del presente (Abu-Zeid y Stanley 1990; Abdel Wahab y Stanley 1991).

Al comparar la argumentación de Hoffmeier con la información geomorfológica disponible y su reconocimiento sobre imágenes satelitales (Fig. 6a), resulta poco probable que al sur de Hebua discurriera algún canal aún desconocido del Nilo, que el autor denomina brazo el-Borg, como de cualquier otro tributario del brazo Pelusiaco. Puesto que este curso hídrico fue el único que recorrió el sector nororiental de Sinai. Asimismo, resulta improbable que la laguna oriental haya sido, durante el Reino Nuevo - unos 3.200 años antes del presente -, un estuario (Véase Figs. 6 a y b).

La existencia de asentamientos fortificados en la franja norte de Sinaí es innegable, aunque esté sujeto a discusión a cuáles de los fuertes nominados en el 'mapa' de Seti I - y dispuestos a lo largo del Camino de Horus corresponde cada uno. La primera de las fortificaciones mencionadas en la fuente documental es Tjaru, la cual puede ser asimilada con cierta confianza con Hebua I, mientras que la segunda -La Casa del León - y la tercera - El Migdol de Menmaatra - permanecen todavia sin ser identificadas con certeza (Oren 1984: 13).

Debido a que Tell el-Borg está situado a 5 $\mathrm{km}$ al sureste de Hebua, Hoffmeier (2004, 2006) propone homologarla con la segunda fortificación del 'mapa', siempre que Hebua II haya funcionado como un asentamiento independiente de Hebua I / Tjaru. Si lo expuesto no fuera correcto, Tell el-Borg deberia ser calificado como tercera fortaleza. No obstante, el autor prefiere proponer que Tell elBorg fue "La Casa del León”, apoyando su argumentación en la existencia del hipotético brazo del Nilo que pasaba en sus proximidades: el brazo el-Borg cuya existencia no puede probarse.

En esa argumentación, la presencia de ese tributario es necesaria para que se cumpla la expectativa de que Tell el-Borg fuera el punto más lejano a lo largo del camino a Siria que podría ser alcanzado por el agua (Gardiner 1920). Asimismo, para el sostenimiento de esa conjetura también es preciso ignorar la existencia de Tell el-Ghaba (Basilico y Lupo 2007; Crivelli 2006) dentro del esquema defensivo construido por Hoffmeier a partir de un posible camino que vinculaba Hebua I-II, Tell el-Borg, Tell elHerr y Kedua. Al mismo tiempo, es importante señalar que la cronologia de Tell el-Herr no permite relacionarlo con los sitios antes mencionados, puesto que su construcción es 
prácticamente simultánea con los niveles de destrucción identificados en Tell el-Borg y Tellel-Ghaba, tal vez por obra de sus mismos destructores.

\section{A modo de síntesis y conclusión}

La representación de la distribución de personajes y de asentamientos fortificados, de cursos fluviales y del litoral marino graficados en el 'mapa' de Seti I puede ser considerada como un producto cultural ambivalente. Por un lado, puede corresponder a una graficación de un hecho administrativo, generado por burócratas especializados que sintetizan y anacronizan acciones históricas dirigidas a dar cuenta de la presencia del estado egipcio frente a los eventuales avances de poblaciones extranjeras en un espacio de frontera. Se trata, al mismo tiempo, de un hecho creativo, puesto que los elementos que la constituyen adquieren sentido y jerarquía iconográfica, independientemente de las referencias geográficas y fácticas que contienen. En este caso se trata más de una forma de percibir y representar el espacio en el marco de una obra ideológicamente construida que un intento realista de representar el espacio geográfico de la frontera oriental de Egipto y describir las acciones militares del estado.

Al aceptar que los elementos iconográficos corresponden a hechos históricos que tuvieron lugar en referencia a un espacio geográfico, se asume que el resultado obtenido es un mapa, entendiendo por tal una forma de representación de una porción de la superficie de la tierra, proyectada sobre un plano y haciendo uso de diferentes escalas en la graficación de los distintos elementos que lo componen. En el presente caso los convencionalismos propios de la cartografia - proyección, simplificación de atributos y escalas - se expresan en la representación del faraón y en su relación con los vencidos, donde las relaciones espaciales concurren a la jeraquización de las figuras y no a conectar referencias geográficas, que parecerian funcionar como elementos subsidiarios de la composición.
No nos interesó preguntarnos aquí acerca de la caracterización de los bordes del antiguo Egipto dentro de un amplio espectro de posibilidades que van desde unas lineas estables, a modo de límites, hasta zonas más indeterminadas de carácter fronterizo. Muy por el contrario, nos propusimos enfocar la figura del faraón como sujeto apto para "hacer", "extender", "estirar" y sobre todo "traspasar" lo que podría ser considerado un límite demarcatorio, una frontera.

Tras las expresiones simbólicas del imperio, la ocupación del norte del Sinai sirvió al acceso por parte del estado egipcio a las fuentes de bienes de prestigio, un tráfico que daba respaldo a la realeza y al orden social, y que puede reconocerse como el reverso de la misma realidad. En esa perspectiva, el rey fue representado como principal protagonista de un papel que aseguraba la disposición de un fondo de poder como centro del sistema retributivo de bienes y prestigio. En esa función, la frontera y su traspaso se constituyeron en los signos visibles de su dominio y poderio, de los que el dios dinástico de Karnak era garante.

Grabado en los muros del gran templo tebano de Amón, el 'mapa' de Seti I muestra que el rey es el siempre victorioso dominador de la periferia, caótico medio exterior, y da así prueba de su poder ordenador a la vez que de su aptitud para operar más allá del cosmos ordenado egipcio. En esta perspectiva, la precisión del diseño del sistema en el que se apoyaban las acciones reales no parece relevante, como tampoco lo sería una descripción realista del paisaje o de las fuerzas militares empleadas en la campaña emprendida por un rey destinado necesariamente al triunfo.

Los relieves militares de Seti I no pueden ser considerados un 'mapa' en tanto representación del espacio geográfico, sino de carácter mítico. Aún cuando el marco de las acciones graficadas guardara relación con los hechos, la narrativa tampoco se propuso ser un registro particularmente verídico de la historia fáctica. La representación del soberano y sus victorias fueron expresivas de su naturaleza esencial. Se trataba de una formulación plástica conceptual más que 
realista, cuyo desarrollo estético respondía a fundamentos ideológicos y no a una intención de lograr un retrato naturalista del rey.

Las representaciones satelitales muestran que el camino hacia el Levante a través de la franja norte de Sinai pasaba por el norte de la laguna oriental y en consecuencia Tell el Borg quedaria a un lado. ¿Cuál era su función en la frontera sinaitica? es un interrogante que por lo menos debería volver a plantearse.

MANZI, L.M.; PEREYRA, M.V. The Sinai frontier of Egypt. From Seti I 'map' to the satellite photography. Revista do Museu de Arqueologia e Etnologia, São Paulo, 20: 293-310, 2010.

Abstract: Geographic information represented in the Seti I's Karnak military reliefs is compared with the geomorphology of northern Sinai, in order to elucidate the New Kingdom iconographic practices and to discuss the recurrent homologation in the bibliography of the archaeological settlements located in the area, with the fortresses mentioned in those monuments

For that purpose, were analyzed the epigraphic records and the geomorphologic unities from satellite images, that masiglos después delde possible: to precise the meaning of the scenes; to establish accurately which were the available terrains during the New Kingdom in that highly dynamic environment; and to discuss the role of the frontier settlements.

Keywords: Iconography - Seti I map - Karnak - Military reliefs - Sinaitic frontier - Geomorphologic unities - Fortified settlements.

\section{Referencias bibliográficas}

ABDEL WAHAB, H.; STANLEY, D.

1991 Clay Mineralogy and the Recent Evolution of the North-Central Nile Delta, Egypt. Journal of Coastal Research, 7 (2): 317-329.

ABU-ZEID, M.; STANLEY, D.

1990 Temporal and Spatial Distribution of Clay Minerals in Late Quaternary Deposits of the NileDelta, Egypt. Journal of Coastal Research, 6 (3): 677-698.

ALTENMÜLlER, H..; MOUSSA, A.M.

1991 Die Inschrift Amenemhets II. aus dem Ptah-Tempel von Memphis. Ein Vorbericht. Studien zur Altägyptischen Kultur, 18: 1-48.

ANGENOT, V.

2002 Discordance entre texte et image. Deux exemples de l'Ancien et du Nouvel Empire. Göttinger Miszellen, 187: 11-22.

\section{BASÍLICO, S.; LUPO, S.}

2007 The final Stage and Abandonment of Tell el-Ghaba, Nort Sinai: a Site on the
Egyptian Eastern Border. In: Goyon, J.; Cardin, Ch. (Eds.) Proceedings of the $9^{\text {th }}$ International Congress of Egyptologists. Vol. 1. Orientalia Lovainiensia Analecta 150. Leuven, Peters: 152-160.

BELOVA. G.

2003 The Eastern Borders of Egypt: New Data. In: Hawass, Z. (Ed.) Egyptology at the Dawn of the Twenty-first Century. Proceedings of the $8^{\text {th }}$ International Congress of Egyptologists. Cairo, 2000. Vol. 2. Cairo, American University: 113-122.

BREASTED, J.H.

1906 Ancient Records of Egypt. Historical Documents, III. The Nineteenth Dynasty. New York: Russell \& Russell.

BROADHURST, C.

1989 An Artistic Interpretation of Sety I's War Reliefs". Journal of Egyptian Archaeology, 75: 229-234. 
BRYAN, B.M.

1996 The Disjunction of Text and Image in Egyptian Art. In: Manuelian, P. der (Ed.) Studies in Honor William Kelly Simpson I. Boston, Museum of Fine Arts, Department of Ancient Egypt, Nubia, and Near Eastern Art: 161-168.

CABROL, A.

2001 Les voies processionnelles. Orientalia Lovainiensia Analecta 97. Leuven: Peeters.

CRIVELLI MONTERO, E.

2006 El extremo oriental del Delta en el pasado y en la actualidad. In: Basílica, S.; Lupo, S. (Eds.) Tell El-Ghaba, N de Sinai, Egipto. Alimentación, producción e intercambio. Buenos Aires, Dunken: 11-31.

DEGRÈVE, A.

2006 La campagne asiatique de l'an 1 de Séthy $1^{\text {er }}$ représentée sur le mur extérieur nord de la salle hypostyle du temple d'Amon a Karnak. Revue d'Égyptologie, 57: 47-76.

EL-SAADY, $\mathrm{H}$.

1992 The Wars of Seti 1 at Karnak: A New Chronological Structure". Studien zur Altägyptischen Kultur, 19: 285-294.

ERMAN, A; GRAPOW, H. (Eds.)

1971 Wörterbuch der ägyptischen Sprache, 5 vols. Berlin; Leipzig: Deutschen Akademie.

FAULKNER, R.O.

1947 The Wars of Sethos 1. Journal of Egyptian Archaeology, 33: 34-39.

1976 A Concise Dictionary of Middle Egyptian. Oxford, Griffith Institute.

GABALLA, O.A.

1976 Narrative in Egyptian Art. Mainz am

GALÁN, J.M. Rhein: Philipp von Zabern.

1995 Victory and Border. Terminology related to Egyptian Imperialism in the XVIII ${ }^{\text {th }}$ Dynasty. Hildesheimer Ägyptologische Beiträge 40. Hildesheim, Gerstenberg.

GARDINER, A.

1920 The Ancient Military Road between Egypt and Palestine. Journal of Egyptian Archaeology, 6: 99-116.

1979 Egyptian Grammar: An Introduction. 3e. Oxford: Griffith Institute.

HOFFMEIER, J.K.

2004 Tell el-Borg on Egypt's Eastern Frontier: A Preliminary Report on the 2002 and 2004 Seasons. Journal of the American Research Center in Egypt, 41: 85-111.

2006 "The Walls of the Ruler" in Egyptian
Literature and the Archaeological Record: Investigating Egypt's Eastern Frontier in the Bronze Age. Bulletin of the American Schools of Oriental Research, 343:1-20.

HOFFMEIER, J.K.; ABDEL MAKSOUD, M.

2003 A New Military Site on 'The Ways of Horus' - Tell el-Borg 1999-2001: A Preliminary Report. Journal of Egyptian Archaeology, 89: 169-197.

\section{KITCHEN, K.A.}

1993 Ramesside Inscriptions, Translated and Annotated: Translations. Vol. I. Oxford: Blackwell.

MARCOLONGO, B.

1992 Évolution du paléo-environnement dans la partie orientale du Delta du Nil depuis la transgression flandrienne (8000 BP) par rapport aux modèles de peuplement anciens. Cahier de Recherches de l'Institut de Papyrologie et d'Egyptologie de Lille, 14: 23-31.

MURNANE, W.J.

1992 The Road to Qadesh: A Historical Interpretation of the Battle Reliefs of King Seti 1 at Karnak. Studies in Ancient Oriental Civilizations 42. Chicago, University of Chicago, Oriental Institute.

OREN, E.

1984 Migdol: A new Fortress on the Edge of the Eastern Nile Delta. Bulletin of American Society of Oriental Research, 256: 7-44.

1987 The "Ways of Horus" in North Sinai. In: Rainey, A. (Ed.) Egypt, Israel, Sinai. Archaeological and Historical Relationships in the Biblical Period. Tel Aviv, Tel Aviv University: 69-119.

QUIRKE, S.

1989 Frontier or border?, the Northeast Delta in the Middle Kingdom texts. In: Proceedings of Colloquium: The archaeology, Geography and History of the Egyptian Delta in Pharaonic Times, Wadham College 29-31 August, 1988, Oxford. Discussions in Egyptology, Special Number 1. Oxford, Cotswold: 261-274.

SCHULMAN, A.R.

1988 Ceremonial Execution and Public Rewards. Some Historical Scenes on New Kingdom Private Stelae. Orbis Biblicus et Orientalis 75. Freiburg: Universitätsverlag Freiburg; Göttingen: van der Hoeck \& Ruprecht.

SNEH, A.; WEISSBROD, T.

1973 Nile Delta: The Defunct Pelusiac Branch Identified. Science, 180: 59-61. 
SPALINGER, A.J.

1979 The Northern Wars of Seti 1: An Integrative Study. Journal of the American Research Center in Egypt, 16: 29-47.

2005 War in Ancient-Egypt: the New Kingdom. Malden, Oxford, Carlton: Blackwell.

STANLEY, D.

1988 Subsidence in the Northeastern Nile Delta: Rapid Rates, Possible Causes, and Consequences. Science, 240 (4851): 497500.

STANLEY, D.; BERNASCONI, M.; JORSTAD, TH.

2008 Pelusium, an Ancient Port Fortress on Egypt's Nile Delta Coast: Its Evolving Environmental Setting from Foundation to Demise. Journal of Coastal Research, 24 (2): 451-462.

STANLEY, D.; WARNE, A.

1993 Nile Delta: Recent Geological Evolution and Human Impact Science, 30 (April: $\mathrm{n}^{\circ}$ 260): 628-634.

1998 Nile Delta in its Destruction Phase. Journal of Coastal Research, 14 (3): 794 825.

STANLEY, D.; WINGERATH, J.

1996 Clay Mineral Distributions to Interpret Nile Cell Provenance and Dispersal: I.
Lower River Nileto Delta Sector. Journal of Coastal Research, 12 (4): 911-929.

TEFNIN, R.

1979 Image et histoire: Refléxions sur l'usage documentaire de l'image égyptienne. Crónique d'Égypte, 54: 218-44.

1984 Discours et iconicité dans l'art égyptien. Göttinger Miszellen, 79: 55-69.

1991 Éléments pour une sémiologie de l'image égyptienne. Crónique d'Égypte, 66: 60-88.

VAN ESSEZ-MERCHEZ, E.

1992 La syntaxe formelle des reliefs et de la grande inscription de l'an 8 de Ramsès III à Medinet Habu. Crónique d'Égypte, 67: 211-239.

VERGNIEUX, R.

1989 L'organisation de l'espace (I): du sacré au profane. Bulletin de la Société d'Égyptologie de Géneve: 165-171.

ZINGARELLI, A.P.

2003 El poder del faraón en los confines del estado. In: Ramos, M.; Néspolo, E. (Eds.) Signos en el tiempo y rastros en la tierra. III Jornadas de Arqueología e Historia de las Regiones Pamapeana y Patagónica. Luján, Universidad Nacional de Luján, Departamento de Ciencias Sociales: 337-345. 